\title{
Encoding of Temporal Probabilities in the Human Brain
}

\author{
Domenica Bueti, ${ }^{1,3}$ Bahador Bahrami, ${ }^{1,2}$ Vincent Walsh, ${ }^{1}$ and Geraint Rees ${ }^{1,2}$ \\ ${ }^{1}$ Institute of Cognitive Neuroscience, University College London, London WC1N 3AR, United Kingdom, ${ }^{2}$ Wellcome Trust Centre for Neuroimaging, \\ University College London, London WC1N 3BG, United Kingdom, and ${ }^{3}$ Neuroimaging Laboratory, Fondazione Santa Lucia, 00179 Rome, Italy
}

Anticipating the timing of future events is a necessary precursor to preparing actions and allocating resources to sensory processing. This requires elapsed time to be represented in the brain and used to predict the temporal probability of upcoming events. While neuropsychological, imaging, magnetic stimulation studies, and single-unit recordings implicate the role of higher parietal and motor-related areas in temporal estimation, the role of earlier, purely sensory structures remains more controversial. Here we demonstrate that the temporal probability of expected visual events is encoded not by a single area but by a wide network that importantly includes neuronal populations at the very earliest cortical stages of visual processing. Moreover, we show that activity in those areas changes dynamically in a manner that closely accords with temporal expectations.

\section{Introduction}

Time frames almost every aspect of human behavior. We rely on the sense of elapsed time to plan actions and anticipate salient environmental events to guide behavior. Successful anticipation of environmental events requires capturing and encoding knowledge about the temporal pattern of sensory stimulation. Little is known about the neural mechanisms underlying the ability to encode temporal expectancies in the human brain. Several neurophysiological studies in monkeys have focused on single cortical sites in either association- or motor-related cortices (Onoe et al., 2001; Leon and Shadlen, 2003; Janssen and Shadlen, 2005; Genovesio et al., 2006). For example, neurons in lateral intraparietal area show anticipatory activity reflecting an internal representation of the elapsed time used to predict the probability that a certain event will occur (Janssen and Shadlen, 2005). However, these studies do not clarify whether this time-dependent activity is a property of an individual cortical region or a shared property of a wider network of areas that have been associated with temporal processing in human studies (Lewis and Miall, 2003).

Neuropsychological studies emphasize the role of the cerebellum and the basal ganglia (Ivry and Keele, 1989; Harrington et al., 1998), whereas neuroimaging studies suggest the involvement of higher-level parietal, premotor, and prefrontal cortices (Coull et al., 2004; Bueti et al., 2008c). Rather less attention has been paid to structures earlier in the visual pathway (Bueti et al., 2008a). It has been suggested, on theoretical grounds, that primary and extrastriate visual cortex may play a key role in encoding temporal variables. According to such a hypothesis, primary visual cortex might allocate salience to retinotopic locations according to

Received May 12, 2009; revised Jan. 13, 2010; accepted Jan. 19, 2010.

This study was supported by the Wellcome Trust (to G.R.), Leverhulme Trust (to D.B.), Graduate School Research Scholarships, Overseas Research Scholarships, UK (to B.B.), and the Royal Society and the Medical Research Council (to V.W.). We thank Dr. Elaine Anderson for helpful comments on this manuscript.

Correspondence should be addressed to Dr. Domenica Bueti, Institute of Cognitive Neuroscience, University College London, 17 Queen Square, London WC1N 3AR, UK. E-mail: domenica.bueti@googlemail.com.

DOI:10.1523/JNEUROSCI.2254-09.2010

Copyright $\odot 2010$ the authors $\quad 0270-6474 / 10 / 304343-10 \$ 15.00 / 0$ statistical inhomogeneities in both the spatial and temporal pattern of retinal input (Barlow, 1990; Schwartz et al., 2007). However, although primary visual cortex responses can be modulated by attention before presentation of a visual stimulus (Kastner et al., 1999; Sharma et al., 2003), in humans no previous study has investigated whether such stimulus-independent signals in early sensory structures can encode the changing temporal expectancy that an external event might occur.

To investigate the neural correlates of temporal expectancy in visual cortex and other cortical structures, 12 participants were trained to anticipate the occurrence of a visual event. On each trial, participants viewed a visual stimulus for several seconds and were instructed to press a key as soon as its color changed from blue to yellow. The timing of the color change was determined by one of two different probability distributions. We examined both neuroimaging and behavioral data for evidence of a neural signature of temporal expectancy.

\section{Materials and Methods}

\section{Subjects}

Twelve right-handed healthy human subjects (four female, mean age 25 years SD 5.2 years) with normal or corrected-to-normal vision gave written informed consent to participate in this study, which was approved by the local ethics committee.

\section{Paradigm}

The visual stimulus was an annulus subtending 12 degrees of visual angle centered on central fixation. Each trial started with the presentation of a blue annulus (the target) that after a variable interval of time (specified by one of two possible probability distributions) changed color from blue to yellow. The color change was the "go" signal. The stimulus luminance for the target and the go signal were respectively 44 and $173 \mathrm{~cd} / \mathrm{m}^{2}$. Background luminance was $3 \mathrm{~cd} / \mathrm{m}^{2}$. The time interval between the presentation of the target and the go signal was a random variable whose probability distribution was fixed throughout a single session. The probability distributions and all the mathematical parameters used were based on previous work in monkey (Janssen and Shadlen, 2005).

We used two probability distributions: unimodal and bimodal. 
The unimodal distribution $U(t)$ of go times was a single Weibull function delayed $(d)$ by $6 \mathrm{~s}$ :

$$
U(t)=\left\{\begin{array}{cl}
3 \alpha(t-d)^{2} e^{-\alpha(t-d)^{3}} & \text { for } t>d \\
0 & \text { otherwise }
\end{array}\right.
$$

$\alpha=0.05$

The bimodal distribution $B(t)$ was the sum of two nonoverlapping Rayleigh distributions, delayed by $d_{1}$ and $d_{2}$, as follows:

$$
B(t)=\frac{1}{2}\left(R_{1}+R_{2}\right)
$$

where

$$
R_{i}=\left\{\begin{array}{cc}
2 \alpha_{i}\left(t-d_{i}\right) e^{-\alpha_{i}\left(t-d_{i}\right)^{2}} & \text { for } t>d_{i} \\
0 & \text { otherwise }
\end{array}\right.
$$

where $\alpha_{1}=0.25, d_{1}=2.50 \mathrm{~s}, \alpha_{2}=0.9, d_{2}=13 \mathrm{~s}$. The go time was drawn with equal probability from $R_{1}$ or $R_{2}$.

Each probability distribution was associated with two different anticipation profiles. Anticipation can be expressed in terms of hazard rate, which is the conditional probability that an event will occur given that it has not yet occurred. This is the probability that the go signal will occur at time $t$ divided by the probability that it has not yet occurred, as described by the following:

$$
h(t)=\frac{f(t)}{I-F(t)},
$$

where $f(t)$ is either $U(t)$ or $B(t)$, and $F(t)$ is the cumulative distribution, as follows:

$$
\int_{0}^{t} f(s) d s .
$$

Based on the assumption that elapsed time is known with uncertainty that scales with time, we calculated "subjective" hazard rates (see also Janssen and Shadlen, 2005). To obtain these, the probability density function $f(t)=U(t)$ or $B(t)$ was first smoothed with a normal distribution whose SD was proportional to elapsed time, as follows:

$$
\tilde{f}(t)=\frac{1}{\phi t \sqrt{2 \pi}} \int_{-\infty}^{\infty} f(t) e^{-(\tau-t)^{2} /\left(2 \phi^{2} t^{2}\right)} d \tau .
$$

The coefficient of variation, $\varphi$, is a Weber fraction for time estimation ( $\varphi=0.21$; Gibbon et al., 1997; Janssen and Shadlen, 2005). Equation 5 implements the idea that participants' estimates of elapsed time carry uncertainty. Thus, an event occurring at objective time $t_{0}$ is perceived as if it occurred at $t_{0} \pm \varphi$. The subjective hazard rate is then obtained by substituting $\tilde{f}(t)$ and its defined integral, $F(t)$, into Equation 3 . We refer to these subjective hazard rates as anticipation functions $A_{\mathrm{u}}$ for the unimodal distribution of go signals and $A_{\mathrm{b}}$ for the bimodal distribution.

The two probability distributions and the associated anticipation functions were used to select the distribution of random go times. We refer to these distributions of random go times as the "time schedule."

The change of the annulus color from blue to yellow represented the go signal, prompting participants to press a key as fast as possible. Participants were instructed to keep track of the elapsed time to predict the occurrence of the go signal and hence prepare to respond to it as fast as possible. Participants were tested on both time schedules (unimodal and bimodal) in separate scanning sessions on different days. Each scanning session was preceded by a behavioral training session held a few days (on average $2 \mathrm{~d}$; range 1-4 d) before scanning. The training session consisted of, on average, 600 trials of the same time schedule. Each scanning session consisted of four runs of 50 trials each (200 trials in total). Six participants performed the unimodal schedule before the bimodal, whereas the remaining six did the opposite.
Functional MRI scanning

A 3 T Siemens Allegra system was used to acquire T2*-weighted echoplanar image (EPI) volumes with blood oxygenation level-dependent (BOLD) contrast. Each EPI volume comprised 32 slices ( $2 \mathrm{~mm}$ axial with an in-plane resolution of $3 \times 3 \mathrm{~mm}$ ) positioned to cover the whole brain. For the main experiment, each run consisted of, on average, 303 volumes (range 271-326). The first five volumes of each run were discarded to allow for T1 equilibration effects. Volumes were acquired continuously with a repetition time (TR) of $2.08 \mathrm{~s}$ per volume. In addition, a T1weighted anatomical image was acquired for each participant.

In six participants, additional fMRI data were collected to identify the boundaries of early retinotopic visual cortex. Two additional runs of 200 EPI volumes were collected using similar coverage and scanning parameters to the main experiment. In each run, participants passively viewed flashing checkerboard patterns (stimulus size was 26 deg in width and 22 deg in height) covering either the horizontal or vertical meridian, alternating with rest periods for 16 epochs of $26 \mathrm{~s}$.

\section{Behavioral data analyses}

Because both anticipation functions could affect the pattern of reaction times (RTs) under either schedule, we ran for each participant and for both schedules a multiple regression analysis incorporating the following potential explanatory variables:

$$
R T(t)=w_{\mathrm{e}}+w_{\mathrm{u}} A_{\mathrm{u}}(t-\tau)+w_{\mathrm{b}} A_{\mathrm{b}}(t-\tau)+\varepsilon .
$$

Here RT are the reaction times; $w_{\mathrm{e}}$ is a constant term; $w_{\mathrm{u}}$ and $w_{\mathrm{b}}$ are the weights for the $A_{\mathrm{u}}$ (unimodal) and $A_{\mathrm{b}}$ (bimodal) anticipation functions, respectively, delayed by time shift $\tau$; and $\varepsilon$ represents noise, which is assumed to be Gaussian with uncertainty derived from the sample means. We sought to explore the contribution of both anticipation functions to participants' RTs under both behavioral schedules, and to investigate the direction of the correlation between response and anticipation functions. On individual $w_{\mathrm{u}}$ and $w_{\mathrm{b}}$ values we ran a 2-by-2 ANOVA with schedule (bimodal/unimodal) and anticipation function $\left(A_{\mathrm{u}} / A_{\mathrm{b}}\right)$ as main factors. A two-sample $t$ test (Bonferroni corrected with $\alpha$ level $=$ $0.05)$ was then performed to compare the $w_{\mathrm{u}}$ and $w_{\mathrm{b}}$ values within each schedule. We tested the hypothesis that under each schedule RTs were better explained by the appropriate anticipation function $\left(A_{\mathrm{u}}\right.$ in unimodal and $A_{\mathrm{b}}$ in bimodal) and that the correlation between RTs and anticipation functions was more negative for the anticipation function appropriate for the schedule. In other words, we predicted that in the unimodal schedule $w_{\mathrm{u}}<w_{\mathrm{b}}$ and in the bimodal schedule $w_{\mathrm{b}}<w_{\mathrm{u}}$.

We first analyzed the behavioral data acquired in the training and in the scanning sessions separately. Because there were no substantial differences comparing the results of these analyses, for each time schedule we then collapsed the data acquired during training and scanning sessions and we performed the analysis on these collapsed data.

\section{Functional MRI data analyses}

Main experiment: whole-brain analysis. Functional imaging data were analyzed using Statistical Parametric Mapping software (SPM2, Wellcome Department of Imaging Neuroscience, University College London). All image volumes were realigned spatially to the first volume and slice time corrected. Resulting volumes were spatially normalized to a standard EPI template volume based on the Montreal Neurological Institute reference brain in the space of Talairach and Tournoux (Talairach and Tournoux, 1988) and resampled to $2 \mathrm{~mm}$ isotropic voxels. The normalized image volumes were then smoothed with an isotropic Gaussian kernel (FWHM $=7 \mathrm{~mm}$ ). The data acquired in the two scanning sessions were analyzed together using an event-related random-effects model. Voxels that were activated in the experimental conditions were identified using a statistical model containing regressors that represented the transient responses evoked by the individual trials in each condition. The event-related changes in evoked activity were modeled by convolving an empirically derived hemodynamic impulse response function with trains of unitary events that were aligned with the trial onsets. Each component of the model served as a regressor in a multiple regression analysis that included the onset of the stimulus target, the onset of the go signal, and the motion correction parameters as effects of no interest. The two an- 
ticipation functions $A_{\mathrm{u}}$ and $A_{\mathrm{b}}$ (as specified by Eq. 5) were instead the variables of interest. The data were high-pass filtered (cutoff frequency $0.0083 \mathrm{~Hz}$ ) to remove low-frequency signal drifts, and global changes in activity were removed by proportional scaling. The resulting parameter estimates for each regressor at each voxel were then entered into a second-level analysis where each participant served as a random effect in a within-participants 2-by-2 ANOVA with schedule (unimodal and bimodal) and anticipation functions $\left(A_{\mathrm{u}}\right.$ and $\left.A_{\mathrm{b}}\right)$ as main factors. Appropriate corrections were made for nonsphericity (Friston et al., 2002) and correlated repeated measures.

We sought to identify brain areas whose activity during the waiting time between target onset and go signal followed the anticipation function associated with the appropriate random go times. These effects were specified by appropriately weighted linear contrasts and determined using the $t$ statistic on a voxel-by-voxel basis. The contrasts used were as follows: $A_{\mathrm{u}}>A_{\mathrm{b}}$ in unimodal time schedule and $A_{\mathrm{b}}>A_{\mathrm{u}}$ in bimodal time schedule to check for areas positively correlated with the anticipation function appropriate to the behavioral schedule. A statistical threshold was used of $P<0.05_{\mathrm{FDR}}$ (Genovese et al., 2002) corrected for multiple comparisons across the entire brain volume.

Onsets of both the target and the go signal were modeled as variables of no interest to ensure that brain responses associated with these events could not confound our analysis. For completeness, we generated SPM contrasts associated with each of these events. These contrast maps were then entered into second-level random-effects analyses, and statistical inferences for each contrast were derived using one-sample $t$ tests. The results of these control analyses are displayed in supplemental Figure 1 (available at www.jneurosci.org as supplemental material).

To independently test for the goodness of the fit between anticipation functions and data, we further analyzed whole-brain activity on those trials from the bimodal schedule where the go signal had appeared at late trial times (after $10 \mathrm{~s}$ ). In these trials there was no change in the stimulus before $9 \mathrm{~s}$. Nevertheless according to our hypothesis, the signal should be highly correlated with expectation in this early part of the trial. The first-level general linear model (GLM) included the onset of the stimulus target, the onset of the go signal, and the motion correction parameters as effects of no interest. Two distinct bimodal regressors each modeling a different part of the bimodal trial were used as effects of interest. The early $A_{\mathrm{b}}$ regressor modeled the expectation of the first $9 \mathrm{~s}$ of the trial, whereas the late $A_{\mathrm{b}}$ the last $10 \mathrm{~s}$. The two bimodal regressors were obtained, splitting the original bimodal anticipation function $A_{\mathrm{b}}$ (as specified by Eq. 5) in two parts, taking the values above and below $9 \mathrm{~s}$. To test the hypothesis that the BOLD response in long trials was correlated with the expectation in the early part of the trial, SPM contrasts associated with the early bimodal regressor were generated for each participant and were entered into a second-level one-sample $t$ test. A statistical threshold was used of $P<0.05_{\mathrm{FDR}}$ (Genovese et al., 2002) corrected for multiple comparisons across the entire brain volume.

For visualization of time course data, the region of interest (ROI) method was used. ROIs were constructed by creating mask volumes for left mid-calcarine sulcus, cerebellar vermis, right supramarginal gyrus, right supplementary motor area, right midfrontal cortex, by taking the peak voxel from each individual subject from within a $10 \mathrm{~mm}$ sphere centered on the group peak co-ordinate. For each ROI, the raw fMRI signal was extracted from the acquired volumes to form a continuous time series. Trial onsets and offsets were marked in this continuous time series and then shifted by three volumes to account for the hemodynamic lag. Trials of different lengths were then aligned to their onset, $z$-transformed, and averaged to give rise to time course for each region of interest in bimodal and unimodal schedule. Importantly, none of the inferential statistics reported in the paper were based on the outcome of this procedure, and extraction of the time course was performed simply to illustrate the results.

Supplementary retinotopic mapping analysis. Data were spatially realigned to the first volume, coregistered to each individual participant's T1 image, and spatially smoothed with a narrow Gaussian kernel of $5 \mathrm{~mm}$ full-width half-maximum. The data were high-pass filtered (cutoff frequency $0.0083 \mathrm{~Hz}$ ) to remove low-frequency signal drifts and then submitted to a within-participant analysis, using a voxelwise GLM that comprised three delayed boxcar waveforms for each scanning run to extract the mean activity evoked by horizontal or vertical meridian stimulation and rest. Freesurfer (http://surfer.nmr.mgh.harvard.edu/) was used to generate inflated and flattened representations of occipital cortex from each participant's T1-weighted structural image. SPM2 was used to generate activation maps from the fMRI data using appropriately weighted linear $t$ contrasts for the comparison of horizontal and vertical meridian stimulation epochs. Six ROIs (left and right V1, V2/V3 dorsal, $\mathrm{V} 2 / \mathrm{V} 3$ ventral) were obtained by delineating the borders between visual areas using the activation patterns from the meridian localizers according to standard definitions (Sereno et al., 1995; Wandell, 1999). In each retinotopic ROI, we next identified voxels that responded to the stimulus in the main interest by selecting two sets of voxels according to their estimated response to stimulus onset from the regression analysis here of the main experiment (see above). Using the $t$ maps for this contrast, strongly visually responsive voxels were defined as those above the 95th centile of voxel responses within the retinotopic ROI, whereas nonresponsive voxels were defined as those below the fifth centile. This permitted us to define subsets of visually responsive and visually nonresponsive voxels for each retinotopic area. Note that the contrast used to define this subset of voxels was independent of those subsequently characterized. Finally, we extracted the parameter estimates from the SPM2 regression analysis for the two anticipation functions $\left(A_{\mathrm{u}}\right.$ and $A_{\mathrm{b}}$ ) in the two behavioral schedules within each ROI for both visually responsive and visually nonresponsive voxels. This procedure allowed us to determine whether expectancy related activity in occipital areas was specific to the portion of cortex selective for the visual stimulus or more widespread. Because there were no significant differences between the left and right hemispheres and between dorsal and ventral V2/V3 (data not shown), for these analyses data were collapsed across hemispheres (for V1 and V2/V3) and across ventral and dorsal regions (for V2/V3). Estimates of activation within each retinotopic ROI were then entered in repeated-measures ANOVAs with anticipation function $\left(A_{\mathrm{u}}\right.$ and $\left.A_{\mathrm{b}}\right)$ and behavioral schedule (unimodal and bimodal) as within-subject factors and the two sets of voxels as between-subjects factor.

\section{Results}

\section{Behavioral analysis}

The time course of temporal anticipation in this task is formalized by the hazard rate, defined as the conditional probability that an event will occur given that it has not yet occurred (see Materials and Methods). To take into account the fact that time estimation follows Weber's law (Gibbon et al., 1997) with the estimation error increasing proportionally to the duration estimated, the standard formulations of the hazard rate were replaced with their temporally blurred versions, which are termed subjective hazard rates or anticipation functions $\left(A_{\mathrm{b}}\right.$ for bimodal and $A_{\mathrm{u}}$ for unimodal; see Eq. 5 in Materials and Methods and also Janssen and Shadlen, 2005). The anticipation functions associated with the two probabilistic time schedules were clearly different (Fig. 1A). When go times (i.e., when the annulus changed color) were drawn from the unimodal distribution, the anticipation function increased monotonically with elapsed time. In contrast, when go times were drawn from the bimodal distribution, the anticipation function was triphasic: it rose, fell, and rose again.

Within each schedule, go RTs showed a clear negative correlation with the relevant anticipation function, demonstrating that participants reliably learned to anticipate the timing of the visual signal under both probabilistic time schedules. Figure $1 B$ shows the data for three individuals plus the group mean. In the unimodal schedule, RTs decreased with longer waiting times. In the bimodal schedule, RTs decreased initially and then rose again for go times that happened to be drawn from the first mode (from 3 to10 s). For go times that were drawn from the second mode of the distribution (from $13 \mathrm{~s}$ onwards) RTs decreased monotonically with increasing go time. Individual reaction time data ac- 
A

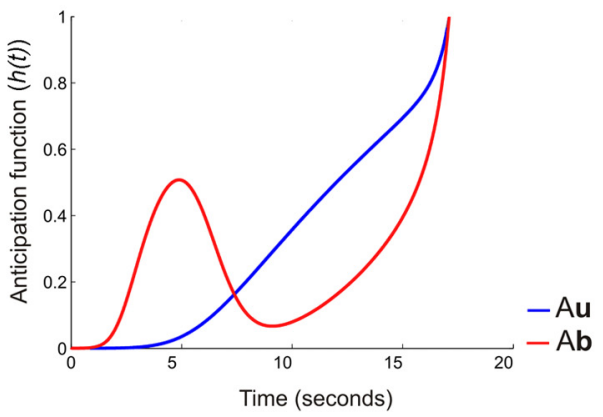

B
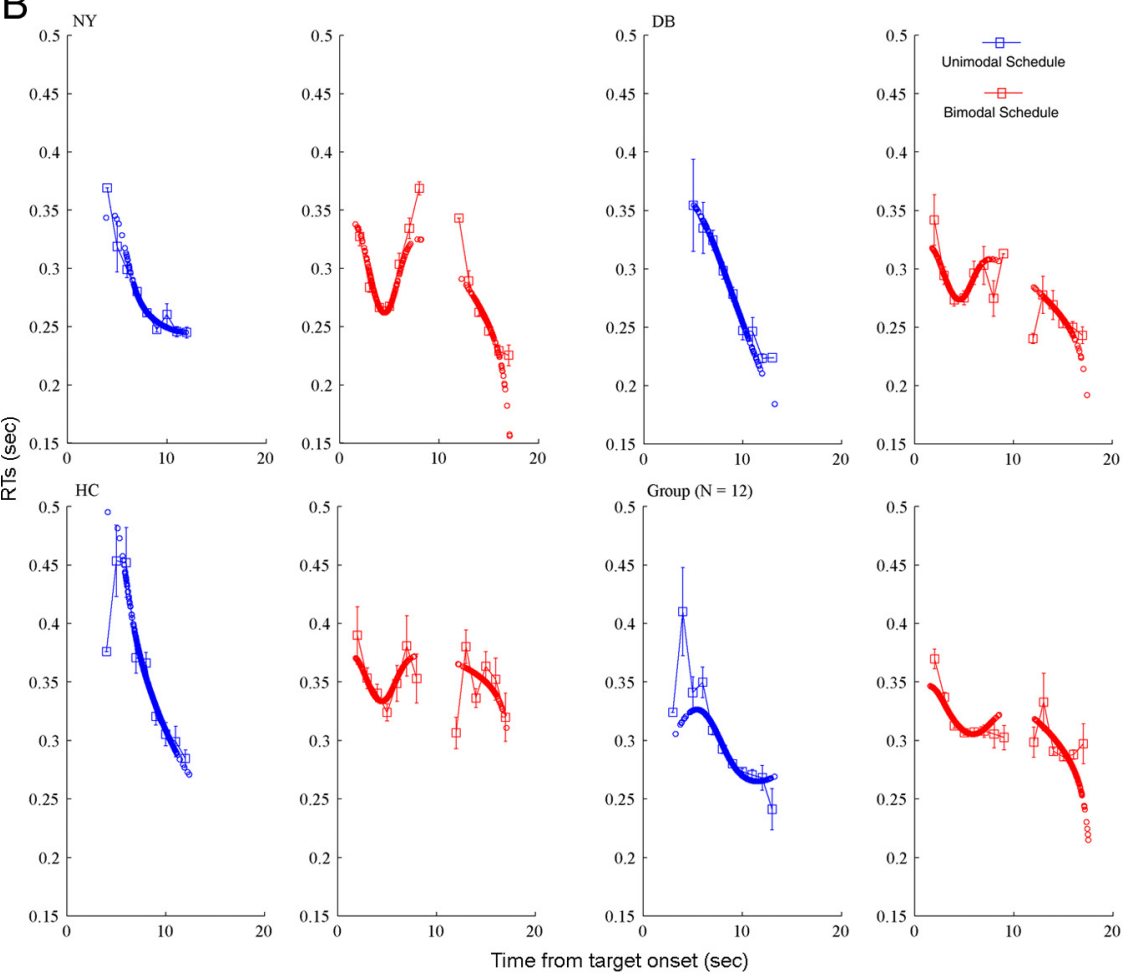

C

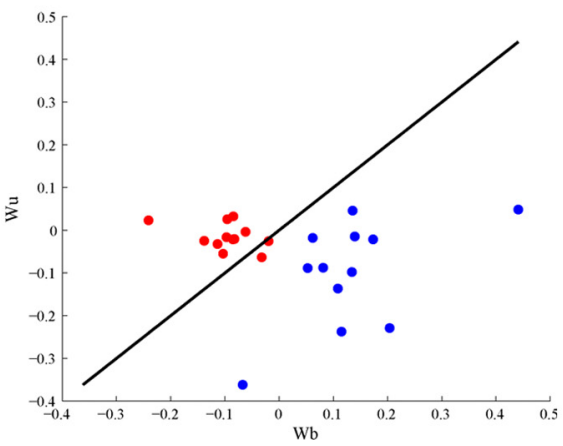

D

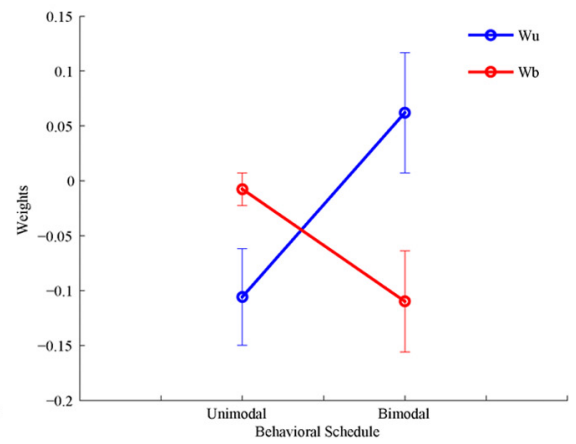

Figure 1. Model of temporal expectancy and behavioral findings. $\boldsymbol{A}$, Subjective hazard rate $(\mathrm{h}(t)$ values, see Eqs. 3 and 5 in Materials and Methods) for the bimodal (red; $A_{b}$ ) and the unimodal (blue; $A_{u}$ ) schedules are plotted as a function of time. $\boldsymbol{B}$, Behavioral data and model predictions are plotted for three example participants and the group $(n=12)$. Each individual graph plots the reaction time as a function of time for the unimodal (blue open squares) and bimodal (red open squares) time schedules. The thick line superimposed on each graph represents the hazard function model obtained by fitting the data to a weighted sum of the two anticipation functions (see Eq. 6 in Materials and Methods). The model predicted an inverse correlation between RTs and the appropriate anticipation function. It is apparent that the model fits the data well, both for individual participants and for the group. $C$, Scatter plot of regression weights for unimodal ( $y$ axis) and bimodal ( $x$ axis) anticipation functions under different anticipation schedules (unimodal schedule: blue, bimodal schedule red). Each dot represents one participant $(n=12)$. Each point represents the pair of regression coefficients obtained by regressing RTs with the two anticipation functions within the same schedule. Thus, red points refer to pairs of $w_{\mathrm{b}}$ and $w_{\mathrm{u}}$ obtained in the bimodal schedule, and blue points refer to $w_{\mathrm{b}}$ and $w_{\mathrm{u}}$ obtained from unimodal schedule (see Eq. 6 in Materials and Methods). D, Mean regression coefficients of 12 participants, separately for bimodal (red) and unimodal (blue) anticipation functions under the unimodal and bimodal timing schedule. The regression quired in both time schedules were fitted by a weighted sum of the two anticipation functions (see Materials and Methods, Eq. 6). The results of the multiple regression (Fig. $1 C, D$ ) showed that participants' reaction times were inversely correlated more strongly with the anticipation function appropriate to the behavioral schedule (interaction schedule by anticipation function $F_{(1,12)}=13,52 P=0.003$; in unimodal schedule $w_{\mathrm{u}}<w_{\mathrm{b}} T_{11}=-6.91 P<$ 0.001 , in bimodal schedule $w_{\mathrm{b}}<w_{\mathrm{u}} T_{11}=$ $-3.8 P<0.001)$. Thus these behavioral data establish that participants were able to represent the passage of time and the time-dependent probability that the go signal was about to occur.

\section{Whole-brain fMRI analysis}

We next analyzed our fMRI data to determine whether they contained a similar signature of temporal anticipation. We hypothesized that neural populations encoding the conditional probability of visual events would show activity better correlated with the unimodal (versus bimodal) anticipation function under the unimodal schedule, but better correlated with the bimodal (versus unimodal) anticipation function under the bimodal schedule. The use of the two anticipation functions as GLM regressors provided quantitative fitting of the two subjective hazard rates (which differed in their temporal profile) to the data, which was, in other words, a measure of how well the appropriate anticipation function fitted the BOLD response.

We also included regressors that modeled (and removed) variance associated with the physical (color) change in the visual stimulus per se (see Materials and Methods).

In the whole-brain analysis, we found that several brain areas demonstrated a pattern of activity correlated with the appropriate anticipation function in the corresponding experimental conditions. Strikingly, the largest cluster $(n=283)$ of significantly activated voxels $(P<0.05$ FDR corrected for multiple comparisons

$\leftarrow$

coefficient associated with unimodal anticipation function $\left(A_{u}\right)$ was more negative under the unimodal than under the bimodal time schedule. The opposite was true for the coefficients associated with bimodal anticipation $\left(A_{\mathrm{b}}\right)$. The plots in $\boldsymbol{C}$ and $\boldsymbol{D}$ show for the group and for the single subject that RTs are better explained by the anticipation function appropriate to the behavioral schedule. These data demonstrate that participants learned to anticipate the go signal in the two time schedules and responded faster when the expectation of the stimulus was higher. 

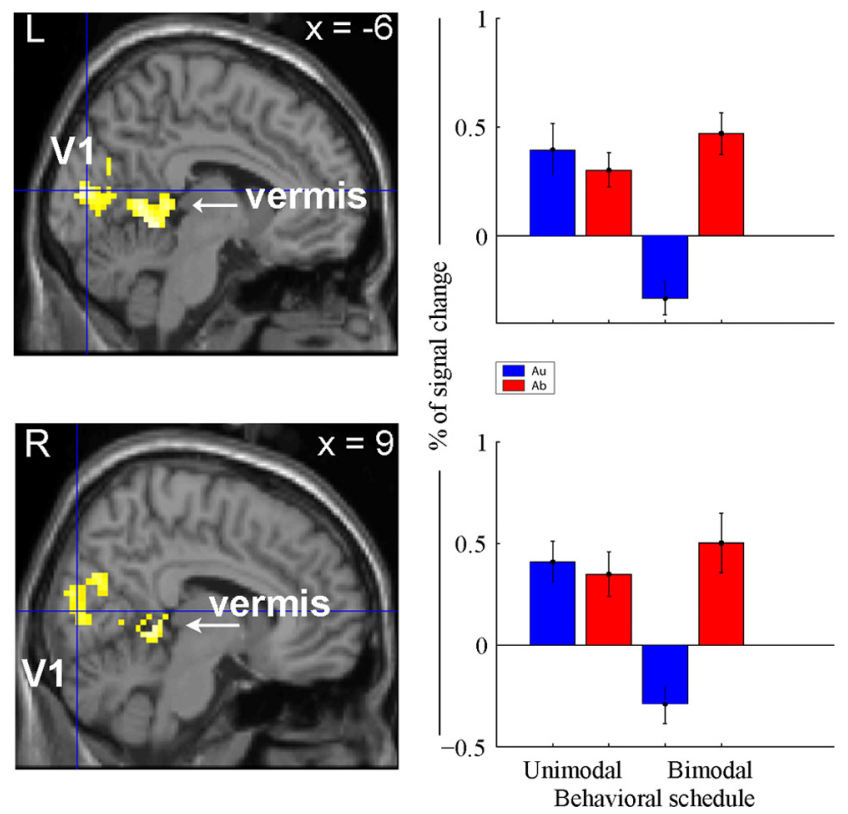

Figure 2. Functional MRI results. Visual areas correlated with the anticipation functions appropriate to the behavioral schedules. The left panel shows group $(n=12)$ peaks of activation in the left and right mid-calcarine sulcus that show significantly greater differences in activity comparing the bimodal (versus unimodal) anticipation functions under the bimodal behavioral schedule with the unimodal (versus bimodal) anticipation functions under the unimodal behavioral schedule. In each case activations are overlaid on a sagittal slice of a template T1-weighted anatomical image in the space of Talairach and Tournoux (see top right of each individual panel for coordinates). Right panels plot the signal change (percent) for unimodal (blue) and bimodal (red) regressors under unimodal or bimodal schedules. Error bars represent SE. In each case it is apparent that there is greater correlation of activity with the unimodal regressors under a unimodal behavioral schedule or the bimodal regressors under a bimodal schedule.

Table 1. Stereotactic brain MNI coordinates (in millimeters) for regions activated in the following contrasts: $A_{u}>A_{b}$ in unimodal time schedule and $A_{b}>A_{u}$ in bimodal time schedule (see Materials and Methods for details)

\begin{tabular}{lccc}
\hline Anatomical structure & $x, y, z$ coordinates $(\mathrm{mm})$ & $Z$ score & Voxels $(n)$ \\
\hline Occipital cortex & & & 283 \\
$\quad$ Mid-calcarine sulcus L & $-6,-84,12$ & 4.21 & \\
$\quad$ Mid-calcarine sulcus R & $9,-84,-9$ & 3.59 & \\
Cerebellum & & & 196 \\
$\quad$ Vermis & $-3,-54,3$ & 4.27 & \\
$\quad$ IV-V lobules L & $-6,-45,-6$ & 4.26 & \\
$\quad$ IV-V lobules R & $9,-45,-3$ & 4.07 & \\
Parietal cortex & & & \\
$\quad$ Supramarginal gyrus R & $57,-42,39$ & 4.23 & 21 \\
Frontal cortex & & & \\
$\quad$ Supplementary motor area R & $6,-21,51$ & 4.24 & 31 \\
$\quad$ Midfrontal R & $45,27,36$ & 3.77 & 12 \\
\hline
\end{tabular}

A statistical threshold of $<0.05_{\mathrm{FDR}}$ (Genovese et al., 2002) corrected for multiple comparisons across the entire brain volume was used.

across the whole brain) was located in the mid-calcarine sulcus bilaterally (left: $-6,-84,12$, peak $z=4.21$; right: $9,-84,-9$, peak $z=3.59$ ) (Fig. 2, Table 1). Both calcarine foci demonstrated relative increases in the signal evoked under the appropriate anticipation function (versus the other function): $A_{\mathrm{u}}>A_{\mathrm{b}}$ in the unimodal schedule and $A_{\mathrm{b}}>A_{\mathrm{u}}$ in the bimodalschedule (Fig. 2, right column). The mid-calcarine foci were not the only brain areas whose response appropriately followed the two anticipation functions in the different experimental conditions. A large cluster (196 voxels) centered on the cerebellar vermis $(-3,-54,3$, peak
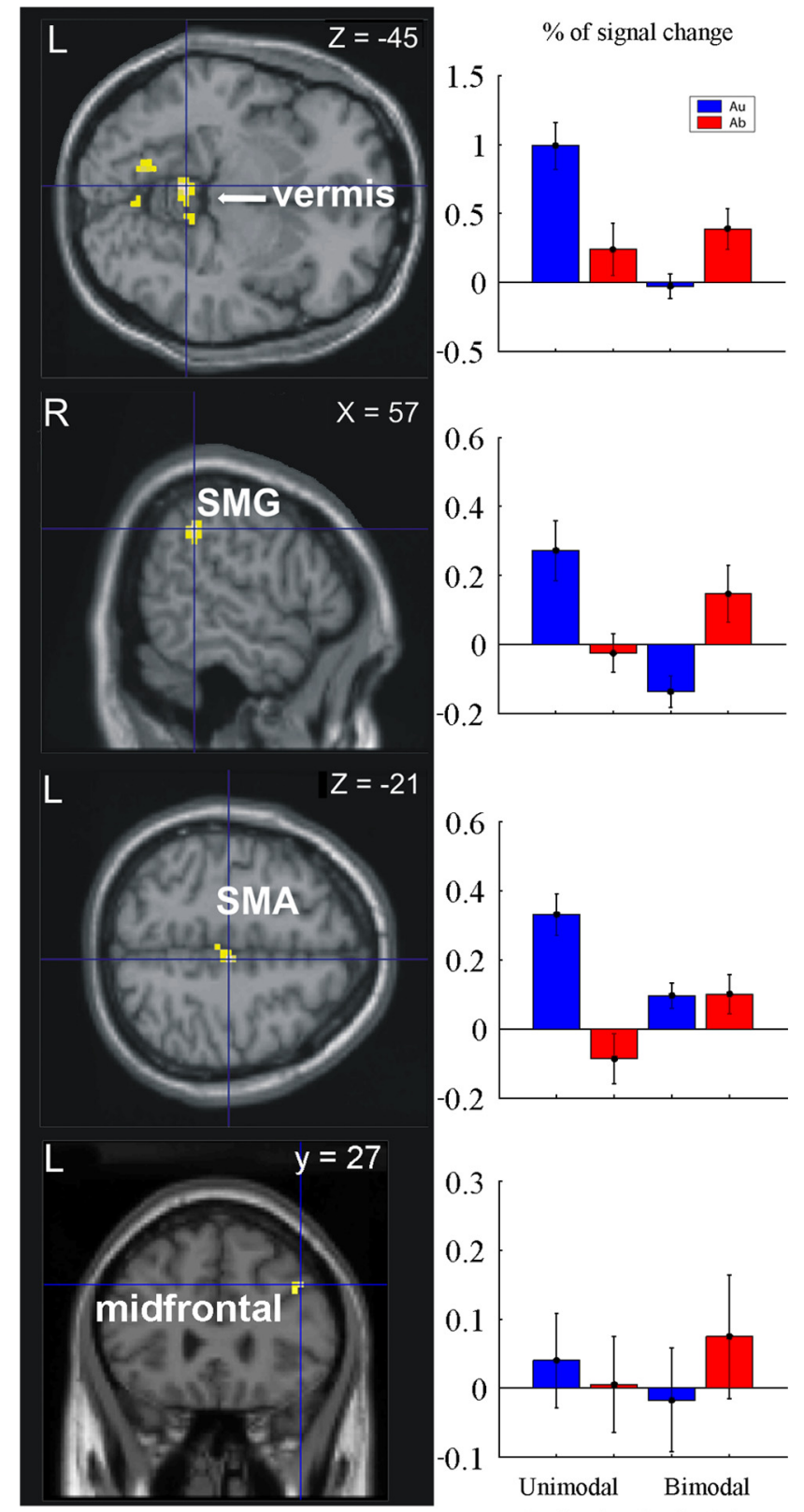

Figure 3. Functional MRI results. Areas correlated with the anticipation functions appropriate to the behavioral schedules. Activation consistent with temporal anticipation was also observed in the cerebellar vermis, right SMG, right SMA, and midfrontal cortex. On the left panel activations are overlaid on a sagittal or axial slice of a template T1weighted anatomical image; the right-hand panels plot the BOLD signal change (percent) for unimodal (blue) and bimodal (red) regressors under unimodal or bimodal schedules. Error bars represent SE.

$z=4.27$ ) including left and right IV and V lobules (according to standard cerebellar taxonomy by Schmahmann et al., 1999; left: $-6,-45,-6$, peak $z=4.26$; right: $9,-45,-3$, peak $z=4.07)$ showed activity significantly correlated $(P<0.05$ FDR corrected $)$ with the anticipation functions (Fig. 3). In addition, right inferior parietal cortex (supramarginal gyrus $\mathrm{SMG}_{\text {nvoxel }=21}: 57,-42,39$, peak $z=4.23)$, right supplementary motor area $\left(\mathrm{SMA}_{\text {nvoxel }=31}\right.$ : $6,-21,51$, peak $z=4.24$ ) and right midfrontal cortex (midfron$\operatorname{tal}_{\text {nvoxel }}=12: 45,27,36$, peak $z=3.77$ ) were also significantly modulated by temporal expectation (Fig. 3 ).

Our analysis procedure explicitly distinguished variability in the signal due to temporal expectation from that due to the visual 
onset of the go signal (see Materials and Methods for details). To confirm the success of this procedure and provide an independent quantitative measure of the goodness of the fit between time courses and the subjective hazard rates, we then analyzed wholebrain activity on only those trials from the bimodal schedule where the go signal had appeared at late trial times (after $10 \mathrm{~s}$ ). For this trial type, there was never any change in the stimulus early in the trial (before $9 \mathrm{~s}$ ), yet our temporal expectation hypothesis predicted that signal should nevertheless be correlated with high expectation early in the trial. To test this hypothesis we used two distinct bimodal regressors modeling expectation of respectively the first (early $A_{\mathrm{b}}$ ) or of the second part of the bimodal trials (late $A_{\mathrm{b}}$ ). Consistent with the hypothesis that a bimodal expectation profile modulated brain activity in bimodal trials independently from the stimulus presentation, we found that activity in V1, vermis, right SMG, SMA, and midfrontal cortex was significantly correlated with the early bimodal regressor (all loci $P<0.05$ FDR-corrected for multiple comparisons; see supplemental Table 1 and supplemental Fig. 2 , available at www.jneurosci.org as supplemental material). This observation converges with our previous analysis to provide compelling evidence that activity in these areas changed as a function of time in accordance with expectations, and that this response was not stimulus-driven.

As an additional qualitative check of these findings we visualized the BOLD time course of those trials from the bimodal schedule where the go signal had appeared at late trial times. Figure 4 shows the time course of the hemodynamic response in the mid-calcarine sulcus, in the vermis, in the right SMG, in the SMA, and in the midfrontal cortex under the unimodal (A) and bimodal (B) schedule. Activity was enhanced when the probability associated with the appearance of the go signal was highest, approximately $\sim 6 \mathrm{~s}$ ( 3 TRs) and $\sim 17 \mathrm{~s}(8 \mathrm{TRs})$ in the bimodal schedule (Fig. 4, right column) and $\sim 10 \mathrm{~s}$ ( 5 TRs) for the unimodal schedule (Fig. 4, left column). Examination of signals in Figure 4 clearly showed that activity in V1, in the vermis, in the right SMG, in the SMA, and in the midfrontal cortex rose early ( $\sim 6 \mathrm{~s}$ or $\sim 3$ TRs) during this bimodal trial type, even though there was no physical change in the visual stimulus at that time.

The decline of the BOLD response observed at the last data point in the unimodal schedule (Fig. 4, left column) was unexpected. According to the unimodal anticipation function, activity in unimodal trials should progressively increase as a function of time. One possible explanation of the observed decay is that the BOLD activity follows the probability of the stimulus rather than the subjective hazard rate (that would fit with the decay of the signal after the point of maximum probability). Alternatively this decay might be an artifact attributable to both the reduced number of trials ending after $10 \mathrm{~s}(<15 \%)$ and the short intertrial interval $(\sim 4 s)$ used. The intertrial interval was kept short to avoid cross-correlation between unimodal and bimodal regressors. It is conceivable that the last data point reflects a time where the task is already finished and participants are no longer expecting the go signal.

\section{Retinotopic analyses}

Probabilistic cytoarchitectonic maps (Eickhoff et al., 2005) confirmed that the visual cortex activation exhibiting activity that correlated with the appropriate hazard function in the group analysis was located in V1 (coordinates for left and right calcarine foci were associated respectively with 80 and $100 \%$ probability of being located in V1). Nevertheless, we next sought to confirm this
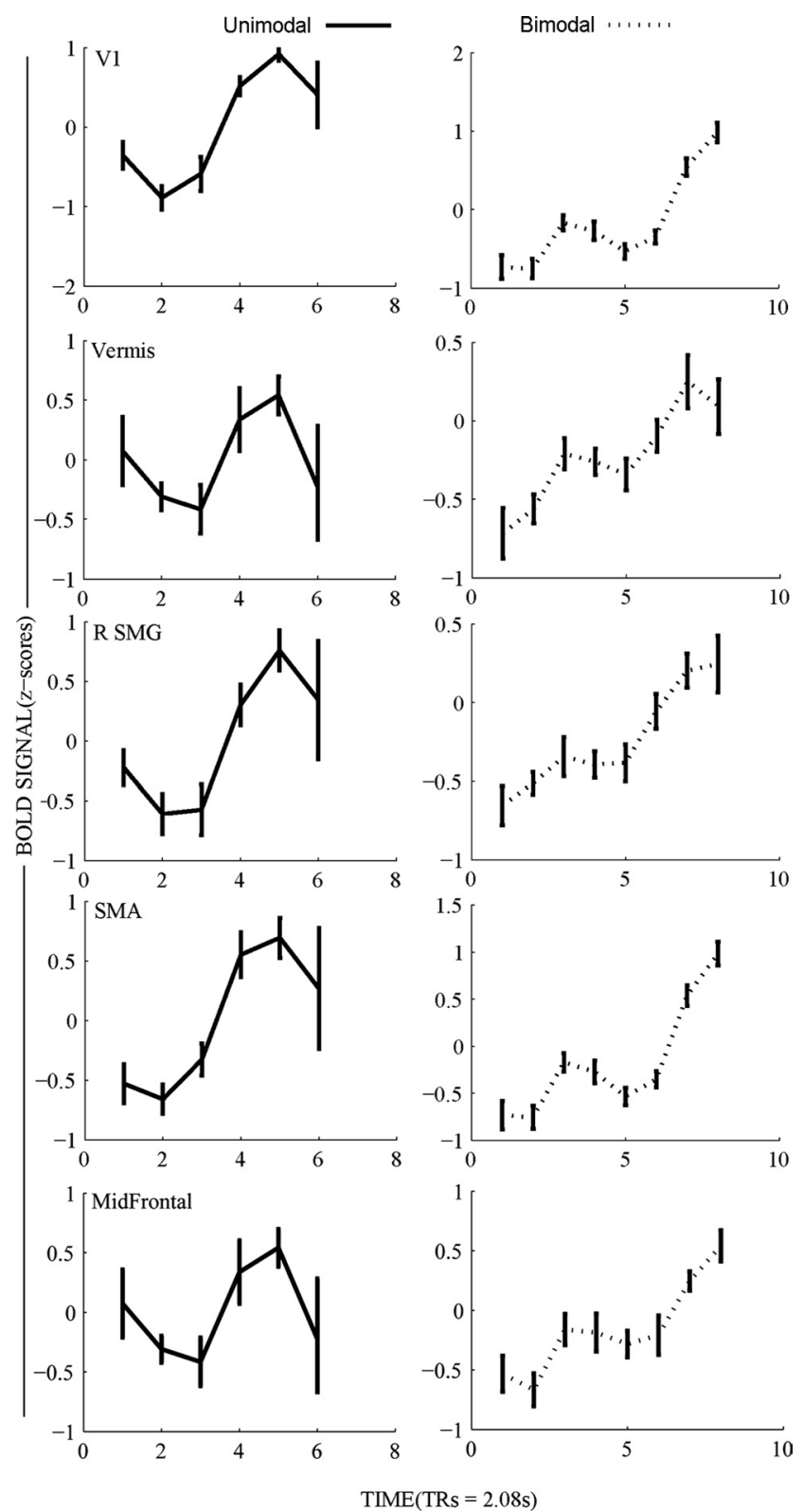

Figure 4. fMRI time course activity. Time course of BOLD contrast activity plotted as a function of time relative to trial onset (time unit is TR $=2.08$ ), for unimodal (continuous line) and bimodal (dotted line) time schedule. For the bimodal schedule only the trial where the go signal occurred at late trial times (after $11 \mathrm{~s}$ ) were considered. Error bars are SEs. See Materials and Methods for details of data analysis. It is apparent that the time course of activity in V1, cerebellar vermis, SMG, SMA, and midfrontal cortex shows a unimodal pattern in unimodal trials and a bimodal pattern in bimodal trials. In particular there is a clear rise in activity early in the bimodal trial even though no physical stimulus change has taken place. This provides convergent evidence that the signal changes we observed represent a neural correlate of temporal expectation unconfounded by signal changes associated with physical changes in the stimulus perse.

finding using retinotopic mapping in individual participants to explore whether the effects we observed were limited to stimulusresponsive regions of $\mathrm{V} 1$, and whether the observed differential activation in medial occipital cortex also encompassed extrastriate visual areas. In a subset of the 12 participants we therefore mapped the visual areas using conventional retinotopic mapping (see Materials and Methods for details). Six ROIs were defined: for left and right V1 and for dorsal and ventral V2/V3 (V2/V3d, $\mathrm{V} 2 / \mathrm{V} 3 \mathrm{v})$. The retinotopic analysis allowed us to test the possibil- 
ity that expectancy related activity in occipital areas might be spatially selective (i.e., restricted to the portion of cortex encoding the annulus). For each participant and in each ROI we identified the amplitude of BOLD response for the two different anticipation functions under the two behavioral schedules in two subsets of voxels: visually responsive to stimulus onset, and visually nonresponsive to stimulus onset (see Materials and Methods for details). Figure $5 A$ shows these responses averaged across the group and collapsed across hemispheres (for V1 and V2/V3 ROIs) and across ventral and dorsal regions (for V2/V3 ROIs). Retinotopic areas V1, V2, and V3 all showed significantly greater BOLD responses for the anticipation function appropriate to the behavioral schedule $\left(A_{\mathrm{u}}>A_{\mathrm{b}}\right.$ in unimodal and $A_{\mathrm{b}}>A_{\mathrm{u}}$ in bimodal). However, this effect was present only in the portions of $\mathrm{V} 1$ and V2/V3 spatially selective for the visual annulus (Fig. 5A, left panel) (spatial selectivity was confirmed by a significant triple interaction of anticipation function by behavioral schedule by sets of voxels: $F_{(1,10)}=8.9, P=0.01$ for $\mathrm{V} 1 ; F_{(1,10)}=26.5, P<$ 0.001 for $\mathrm{V} 2 / \mathrm{V} 3)$. This retinotopic specificity was also consistent with inspection of the activation profile in the relevant contrast ( $A_{\mathrm{u}}>A_{\mathrm{b}}$ in unimodal and $A_{\mathrm{b}}>A_{\mathrm{u}}$ in bimodal schedule), overlaid on individual retinotopic maps. Activations in visual areas were limited to the retinotopic area stimulated by the annulus (Fig. 5A,B).

\section{Discussion}

Together, our findings indicate that in humans, temporal information used to make predictions about behaviorally relevant visual events is encoded not in a single area but in a network of distributed cortical loci. Moreover, these data represent the first evidence of retinotopically specific signals reflecting temporal expectancies in human primary visual cortex and extrastriate visual areas $\mathrm{V} 2 / \mathrm{V} 3$. We observed temporal modulation along the pathway that brings incoming information from primary sensory to motor areas through a visuomotor integrative region represented by the parietal cortex. In nonhuman primates, neurons encoding temporal expectation can be found in lateral intraparietal cortex (Leon and Shadlen, 2003; Janssen and Shadlen, 2005) and area V4 (Ghose and Maunsell, 2002). Such observations are consistent with our finding that neuronal populations in inferior parietal cortex and extrastriate areas showed signal modulations in accord with the anticipation functions. However, our new data go beyond this earlier work by showing that the amplitude of signals from neuronal populations at the earliest stages of cortical processing in human primary visual cortex are already tightly locked to temporal expectation in the absence of any stimulus changes (Figs. 2 and 3; see also supplemental Fig. 2, available at www.jneurosci.org as supplemental material).

Our results demonstrate a relationship between BOLD signals and subjective hazard rate. However, we cannot rule out the possibility that the neural responses we observed follow the actual stimulus probability more or less closely than the subjective estimation of the stimulus probability (i.e., subjective likelihood or subjective hazard rate). Indeed, the actual and the subjective hazard rates were too closely correlated to be used as separate regressors in our fMRI model. We found that the behavior of our participants was significantly modulated by the subjective hazard rate (Fig. $1 B$ ), so it is therefore reasonable to suggest that the neural responses we observed might reflect motor preparation processes. This might in turn raise the question of whether such neural responses might therefore be correlated with the actual motor response. However, we can rule out the possibility that the

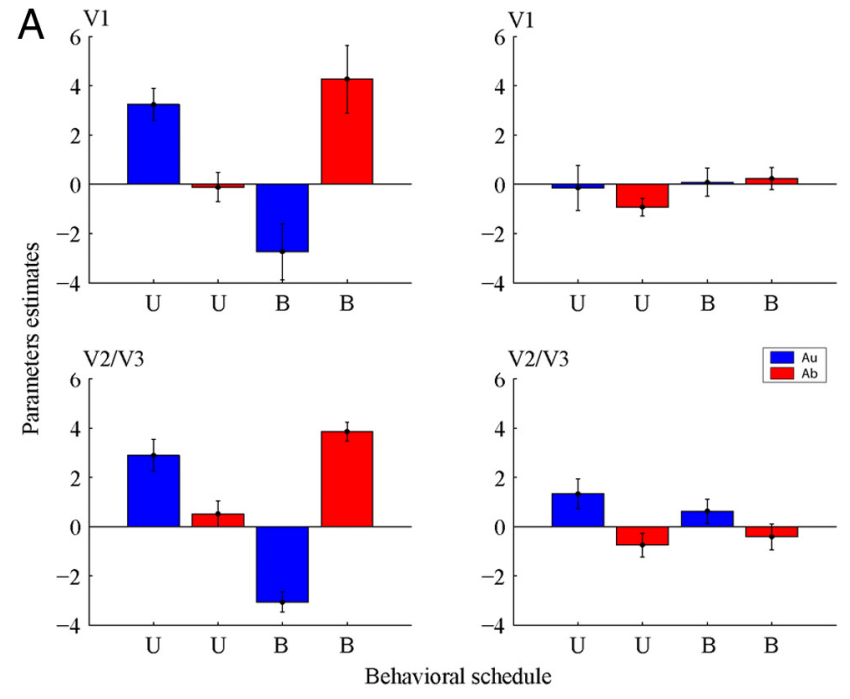

Stimulus responsive voxels Stimulus unresponsive voxels

\section{B}

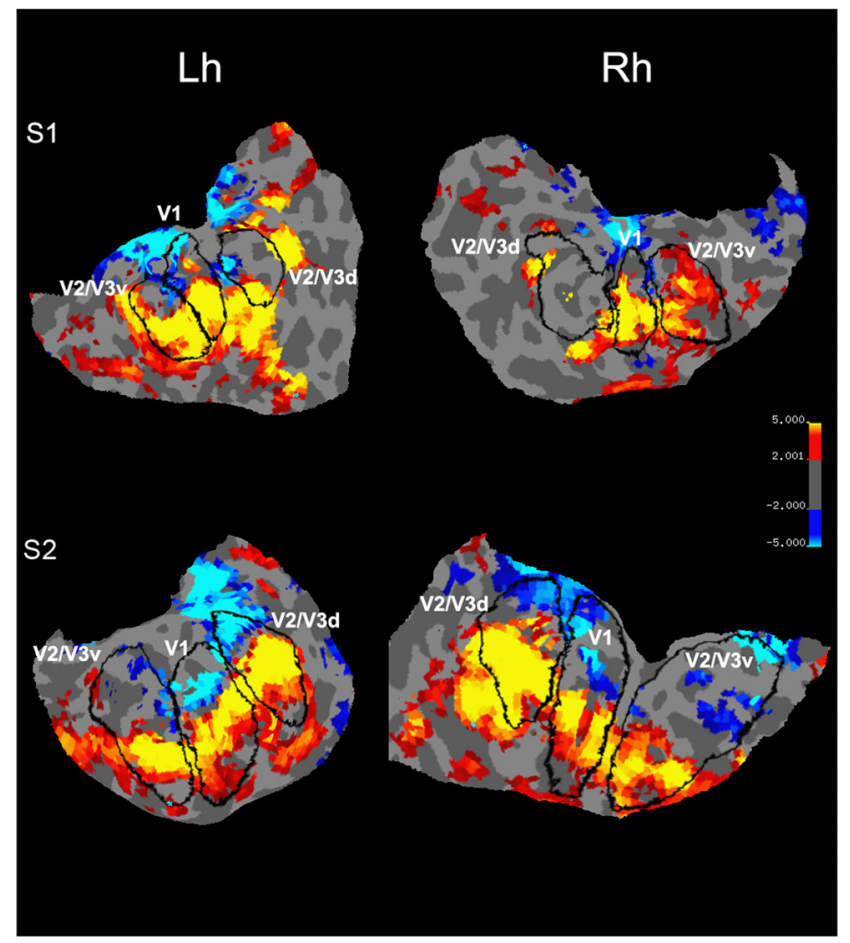

Figure 5. Retinotopic analyses. A, Activation patterns for the six participants for whom retinotopic mapping data were obtained (see Materials and Methods for full details). The panels show the percentage change in B0LD V1 and V2/V3 associated with either unimodal (blue bars) or bimodal (red bars) regressors in two behavioral schedules (U, Unimodal; $\mathrm{B}$, bimodal). In both $\mathrm{V} 1$ and V2/V3 significant expectancy related effects $\left(A_{\mathrm{u}}>A_{\mathrm{b}}\right.$ in unimodal schedule and $A_{\mathrm{b}}>A_{\mathrm{u}}$ in bimodal schedule) were observed only for the voxels that were spatially selective for the stimulus (left, $P=0.01$ and $P=0.02$ for respectively unimodal and bimodal behavioral schedule in $\mathrm{V} 1$; and $P=0.045$ and $P<0.001$ for unimodal and bimodal behavioral schedule in V2/V3). No significant differences between the two anticipation functions were observed for voxels that were spatially unrelated to the stimulus (right). Error bars represent SE. $\boldsymbol{B}$, Activity correlated with temporal anticipation in retinotopic visual maps. SPM maps of activity in the contrast $A_{\mathrm{u}}>A_{\mathrm{b}}$ in the unimodal behavioral schedule and $A_{\mathrm{b}}>A_{\mathrm{u}}$ in the bimodal behavioral schedule overlaid on the flattened retinotopic map of two representative participants (S1-S2). Left-hand panels show the left occipital cortex (Lh), right-hand panels show the right occipital cortex (Rh). V1, V2/V3d, V2/V3v borders (in black) are defined based on standard retinotopic mapping procedures (Sereno et al., 1995). 
actual motor response (i.e., RTs) is the sole factor driving the BOLD signals we observed. First, we found modulation of neural responses associated with temporal expectation early in long bimodal trials, at a time when there was no visual stimulation and no motor response (see Results; also see supplemental Fig. 2, available at www.jneurosci.org as supplemental material). In addition, our observations of expectancy-related activity not only in motor-related brain regions, like premotor and parietal cortex, but also in early visual regions, make an explanation in terms of motor responses alone less likely.

In humans, shifts in the baseline activity of primary visual cortex in the absence of any visual stimulation have been observed (Kastner et al., 1999; Haynes et al., 2005; Silver et al., 2007). This activity is sustained during the time delay preceding stimulus onset, is associated with the deployment of covert spatial attention, and regulated by parietal structures (Chawla et al., 1999; Haynes et al., 2005). In the present study we did not explicitly manipulate spatial attention (i.e., spatial expectancy) but instead required participants to focus on temporal expectation. In contrast to earlier studies, our new findings now show that in the absence of a stimulus, V1 activity reflected temporal expectancy; and critically, such modulation had temporal dynamics that were closely locked to the subjective likelihood that a stimulus will occur. As with spatial expectancy, we found that temporal expectancy-related activity in retinotopic visual cortex was specific to the retinotopic location of the expected stimulus (Fig. 5). However, there were also important differences from previous studies of spatial attention. Specifically, we did not observe any difference in the amplitude of the BOLD response comparing parietal, premotor cortex, and visual areas (Kastner et al., 1999). Moreover, the parietal activation associated with temporal expectancy that we observed was not located in the intraparietal sulcus, like most studies on covert orienting of spatial attention report (Corbetta and Shulman, 2002; Sylvester et al., 2009). Rather, it was centered on the inferior parietal lobule, a location often associated with temporal processing (Buhusi and Meck, 2005; Bueti and Walsh, 2009).

In humans, mechanisms of attentional orienting to time have been investigated with neuroimaging and electrophysiological methods (Nobre et al., 2007). These studies emphasize the effects of temporal predictability on motor preparation and response selection processes. Orienting attention to time has no effect on early ERP components (P100) of target analysis (Griffin et al., 2002; Doherty et al., 2005) and is correlated with the activation of premotor and parietal regions of the left hemisphere (Coull and Nobre, 1998). An important difference between this earlier work and the present study is that the temporal distribution of events in our task was continuous rather than binary (short versus long in Coull and Nobre, 1998) or fixed according to a rhythm (Doherty et al., 2005). The presence of such variability, in our experimental paradigm, may have induced participants to focus more on the temporal aspects of the task rather than on the preparation of the response. The accurate representation of the elapsed time was indeed very relevant for the efficiency of the prediction, as shown by our behavioral results (Fig. $1 \mathrm{~B}$ ). Moreover, in contrast to these previous studies, our data now demonstrate that temporal expectations affect activity not only in parietal and premotor regions but also in retinotopic visual cortex, including primary visual cortex.

It has been suggested (Barlow, 1990; Schwartz et al., 2007) and recently emphasized (Schwartz et al., 2007) that one of the computational roles of the primary visual cortex is the allocation of salience to image locations based on statistical inhomogeneities in space or time. Although several studies have demonstrated such a role for V1 in the spatial domain (Knierim and van Essen, 1992; Kapadia et al., 1995), our results now provide physiological evidence for a representation of temporal statistics of the visual environment in V1. Consistent with this, V1 in rats can show sustained responses after stimulus presentation reflecting two different reward timings (Shuler and Bear, 2006). The role of sensory areas in temporal processing is a very recent finding (Bueti et al., 2008b) and indeed is the subject of some debate (Walsh, 2003; Ivry and Schlerf, 2008). Transient disruption of V5/MT can affect temporal discrimination of visual durations (Bueti et al., 2008a), providing some converging evidence for a role of visual cortex in temporal processing. The present findings now demonstrate the existence of a temporal signal much earlier in the visual pathway and in the primary visual cortex and show that this modulation here is retinotopic specific. This finding also accords with purely psychophysical data in humans (Johnston et al., 2006; Kanai et al., 2006), showing that the apparent duration of a visual stimulus can be selectively distorted in a spatially restricted region of the visual field, suggesting the presence of retinotopically specific mechanisms that can encode temporal information. The existence of a temporal modulation of signals in extrastriate areas V2/V3 is consistent with two psychophysical studies showing the effects of saccadic eye movements on the subjective representation of time (Morrone et al., 2005; Burr et al., 2007). These two studies, together with a theoretical model (Walsh, 2003), suggest the involvement of areas of the visual dorsal stream, important for spatial representations and visuomotor transformations, in temporal encoding networks. Together with our finding that primary and secondary visual cortex can encode time-dependent changes in stimulus expectation, we tentatively propose the presence in theses areas of local temporal mechanisms enabling the computation of elapsed time when visual information is task relevant.

Cerebellum, parietal cortex, SMA, and prefrontal cortex are all implicated in aspects of timing (Spencer et al., 2003; Coull et al., 2004; Vallesi et al., 2007; Bueti et al., 2008c), but nevertheless their specific functional roles concerning temporal processing are far from clear. Our data showing similar types of temporal modulation in accordance with the appropriate anticipation function may suggest that elapsed time is represented in areas wherever this information is needed (Onoe et al., 2001; Ghose and Maunsell, 2002; Leon and Shadlen, 2003; Janssen and Shadlen, 2005). Timing signals in visual (V1, V2/V3, our data; V4, Ghose and Maunsell, 2002; V5, Bueti et al., 2008a,c), visuomotor (parietal cortex), and motor (SMA, cerebellum) areas may all reasonably contribute to both the representation of an expected stimulus and the efficiency with which one can react to it. The striking correspondence between the response patterns of BOLD signals associated with temporal expectancy we observed in humans and patterns of single neuron firing in monkeys (Janssen and Shadlen, 2005) is consistent with a specific computational model of time processing (Durstewitz, 2003). This model originates from the observation that during tasks where animals have to hold a response for predictable amount of time (i.e., working memory, behavioral conditioning, reward timing tasks), neural activity in cortical (prefrontal, parietal, and premotor cortices) and subcortical (thalamus) brain areas, increases slowly and linearly during the delay period and reaches a maximum when choice stimuli or the expected stimulus/reward appears. According to this model, temporal information is embedded in the slowly climbing anticipatory activity of neurons (Durstewitz, 2004). The temporal modu- 
lation of the BOLD response between target onset and go signal observed here could be considered the homolog in humans of the same anticipatory "slowly rising" delay activity observed in monkeys.

Nevertheless, although our data show a widespread representation of temporal information in the human brain, they do not rule out the possibility that such information is only generated in a subset of these areas with specialization for temporal processing (e.g., the cerebellum), and passed on to the network. This represents an intermediate view between centralized (Allan, 1979; Treisman et al., 1990) and distributed (Ivry, 1996; Karmarkar and Buonomano, 2007) temporal mechanisms in which there is some devolution of timing modules. Our data do not distinguish these possibilities, but set out candidate structures whose causal involvement in temporal expectation can now be probed using techniques that transiently disrupt cortical function such as transcranial magnetic stimulation (Alexander et al., 2005; Bueti et al., 2008a,b).

In conclusion, our data show that humans are able to learn features of probabilistic time schedules for changes to a simple visual stimulus. If interpreted in the context of temporal processing, these findings represent the first evidence of the involvement of primary visual cortex and extrastriate areas $\mathrm{V} 2 / \mathrm{V} 3$ in temporal coding. In the alternative context of an attentional interpretation, our data show for the first time that attentional mechanisms can be dynamically time locked to expectations and that this temporal modulation affects very early stages of visual processing.

\section{References}

Alexander I, Cowey A, Walsh V (2005) The right parietal cortex and time perception: back to Critchley and the Zeitraffer phenomenon. Cogn Neuropsychol 22:306-315.

Allan LG (1979) The perception of time. Percept Psychophys 26:340-354. Barlow HB (1990) Vision: coding and efficiency. New York: Cambridge UP. Bueti D, Walsh V (2009) The parietal cortex and the representation of time, space, number and other magnitudes. Philos Trans R Soc Lond B Biol Sci 364:1831-1840.

Bueti D, Bahrami B, Walsh V (2008a) Sensory and association cortex in time perception. J Cogn Neurosci 20:1054-1062.

Bueti D, van Dongen EV, Walsh V (2008b) The role of superior temporal cortex in auditory timing. PLoS One 3:e2481.

Bueti D, Walsh V, Frith C, Rees G (2008c) Different brain circuits underlie motor and perceptual representations of temporal intervals. J Cogn Neurosci 20:204-214.

Buhusi CV, Meck WH (2005) What makes us tick? Functional and neural mechanisms of interval timing. Nat Rev Neurosci 6:755-765.

Burr D, Tozzi A, Morrone MC (2007) Neural mechanisms for timing visual events are spatially selective in real-world coordinates. Nat Neurosci $10: 423-425$.

Chawla D, Rees G, Friston KJ (1999) The physiological basis of attentional modulation in extrastriate visual areas. Nat Neurosci 2:671-676.

Corbetta M, Shulman GL (2002) Control of goal-directed and stimulusdriven attention in the brain. Nat Rev Neurosci 3:201-215.

Coull JT, Nobre AC (1998) Where and when to pay attention: the neural systems for directing attention to spatial locations and to time intervals as revealed by both PET and fMRI. J Neurosci 18:7426-7435.

Coull JT, Vidal F, Nazarian B, Macar F (2004) Functional anatomy of the attentional modulation of time estimation. Science 303:1506-1508.

Doherty JR, Rao A, Mesulam MM, Nobre AC (2005) Synergistic effect of combined temporal and spatial expectations on visual attention. J Neurosci 25:8259-8266.

Durstewitz D (2003) Self-organizing neural integrator predicts interval times through climbing activity. J Neurosci 23:5342-5353.

Durstewitz D (2004) Neural representation of interval time. Neuroreport 15:745-749.

Eickhoff SB, Stephan KE, Mohlberg H, Grefkes C, Fink GR, Amunts K, Zilles
K (2005) A new SPM toolbox for combining probabilistic cytoarchitectonic maps and functional imaging data. Neuroimage 25:1325-1335.

Friston KJ, Glaser DE, Henson RN, Kiebel S, Phillips C, Ashburner J (2002) Classical and Bayesian inference in neuroimaging: applications. Neuroimage 16:484-512.

Genovese CR, Lazar NA, Nichols T (2002) Thresholding of statistical maps in functional neuroimaging using the false discovery rate. Neuroimage 15:870-878.

Genovesio A, Tsujimoto S, Wise SP (2006) Neuronal activity related to elapsed time in prefrontal cortex. J Neurophysiol 95:3281-3285.

Ghose GM, Maunsell JH (2002) Attentional modulation in visual cortex depends on task timing. Nature 419:616-620.

Gibbon J, Malapani C, Dale CL, Gallistel C (1997) Toward a neurobiology of temporal cognition: advances and challenges. Curr Opin Neurobiol 7:170-184.

Griffin IC, Miniussi C, Nobre AC (2002) Multiple mechanisms of selective attention: differential modulation of stimulus processing by attention to space or time. Neuropsychologia 40:2325-2340.

Harrington DL, Haaland KY, Hermanowicz N (1998) Temporal processing in the basal ganglia. Neuropsychology 12:3-12.

Haynes JD, Tregellas J, Rees G (2005) Attentional integration between anatomically distinct stimulus representations in early visual cortex. Proc Natl Acad Sci U S A 102:14925-14930.

Ivry RB (1996) The representation of temporal information in perception and motor control. Curr Opin Neurobiol 6:851-857.

Ivry RB, Keele SW (1989) Timing functions of the cerebellum. J Cogn Neurosci 1:136-152.

Ivry RB, Schlerf JE (2008) Dedicated and intrinsic models of time perception. Trends Cogn Sci 12:273-280.

Janssen P, Shadlen MN (2005) A representation of the hazard rate of elapsed time in macaque area LIP. Nat Neurosci 8:234-241.

Johnston A, Arnold DH, Nishida S (2006) Spatially localized distortions of event time. Curr Biol 16:472-479.

Kanai R, Paffen CL, Hogendoorn H, Verstraten FA (2006) Time dilation in dynamic visual display. J Vis 6:1421-1430.

Kapadia MK, Ito M, Gilbert CD, Westheimer G (1995) Improvement in visual sensitivity by changes in local context: parallel studies in human observers and in V1 of alert monkeys. Neuron 15:843-856.

Karmarkar UR, Buonomano DV (2007) Timing in the absence of clocks: encoding time in neural network states. Neuron 53:427-438.

Kastner S, Pinsk MA, De Weerd P, Desimone R, Ungerleider LG (1999) Increased activity in human visual cortex during directed attention in the absence of visual stimulation. Neuron 22:751-761.

Knierim JJ, van Essen DC (1992) Neuronal responses to static texture patterns in area V1 of the alert macaque monkey. J Neurophysiol 67:961980.

Leon MI, Shadlen MN (2003) Representation of time by neurons in the posterior parietal cortex of the macaque. Neuron 38:317-327.

Lewis PA, Miall RC (2003) Distinct systems for automatic and cognitively controlled time measurement: evidence from neuroimaging. Curr Opin Neurobiol 13:250-255.

Morrone MC, Ross J, Burr D (2005) Saccadic eye movements cause compression of time as well as space. Nat Neurosci 8:950-954.

Nobre A, Correa A, Coull J (2007) The hazards of time. Curr Opin Neurobiol 17:465-470.

Onoe H, Komori M, Onoe K, Takechi H, Tsukada H, Watanabe Y (2001) Cortical networks recruited for time perception: a monkey positron emission tomography (PET) study. Neuroimage 13:37-45.

Schmahmann JD, Doyon J, McDonald D, Holmes C, Lavoie K, Hurwitz AS, Kabani N, Toga A, Evans A, Petrides M (1999) Three-dimensional MRI atlas of the human cerebellum in proportional stereotaxic space. Neuroimage 10:233-260.

Schwartz O, Hsu A, Dayan P (2007) Space and time in visual context. Nat Rev Neurosci 8:522-535.

Sereno MI, Dale AM, Reppas JB, Kwong KK, Belliveau JW, Brady TJ, Rosen BR, Tootell RB (1995) Borders of multiple visual areas in humans revealed by functional magnetic resonance imaging. Science 268:889-893.

Sharma J, Dragoi V, Tenenbaum JB, Miller EK, Sur M (2003) V1 neurons signal acquisition of an internal representation of stimulus location. Science 300:1758-1763. 
Shuler MG, Bear MF (2006) Reward timing in the primary visual cortex. Science 311:1606-1609.

Silver MA, Ress D, Heeger DJ (2007) Neural correlates of sustained spatial attention in human early visual cortex. J Neurophysiol 97:229237.

Spencer RM, Zelaznik HN, Diedrichsen J, Ivry RB (2003) Disrupted timing of discontinuous but not continuous movements by cerebellar lesions. Science 300:1437-1439.

Sylvester CM, Shulman GL, Jack AI, Corbetta M (2009) Anticipatory and stimulus-evoked blood oxygenation level-dependent modulations related to spatial attention reflect a common additive signal. J Neurosci 29:10671-10682.
Talairach J, Tournoux P (1988) Co-planar stereotaxic atlas of the human brain. New York: Thieme Medical.

Treisman M, Faulkner A, Naish PL, Brogan D (1990) The internal clock: evidence for a temporal oscillator underlying time perception with some estimates of its characteristic frequency. Perception 19:705-743.

Vallesi A, Shallice T, Walsh V (2007) Role of the prefrontal cortex in the foreperiod effect: TMS evidence for dual mechanisms in temporal preparation. Cereb Cortex 17:466-474.

Walsh V (2003) A theory of magnitude: common cortical metrics of time, space and quantity. Trends Cogn Sci 7:483-488.

Wandell BA (1999) Computational neuroimaging of human visual cortex. Annu Rev Neurosci 22:145-173. 